\title{
At the Forefront
}

Vic Suller from the Daresbury Laboratory, UK, reporting on the Fifth European Particle Accelerator Conference (Sitges; 10 - 14 June 1996), found that Europe remains at the forefront in most aspects of accelerator technology and its applications.

The advanced nature of European accelerators operating at the boundaries of present day technology was the main feature of the first session of this year's European Particle Accelerator Conference (EPAC'96, the fifth in the series). Y. Petroff (ESRF, Grenoble) described how the European Synchrotron Radiation Facility's 6 $\mathrm{GeV}$ third-generation source would soon be operating with a source brightness which was 100 times higher than the original specification, thanks to improvements in insertion devices and by exceptional control of the accelerator performance. This opens exciting opportunities in a range of science topics as diverse as astrophysics to materials technology. The performance and range of facilities of DESY's HERA collider in Hamburg have also been extended (W. Bialowons, DESY). To produce $820 \mathrm{GeV}$ protons for collision with 30 $\mathrm{GeV}$ positrons, six pre-accelerators are employed. A $20 \%$ increase in the integrated luminosity (to $15 \mathrm{pb}^{-1}$ ) is expected for 1996, and the experimental facilities will be fully exploited by operating the 4 th interaction region. The role of polarization is crucial and is used in the internal gas-jet targets and the longitudinally polarized (70\%) positrons. D. Boussard (CERN, Geneva) described the energy upgrade of CERN's LEP to $90 \mathrm{GeV}$ per beam (well beyond the $\mathrm{W}$-pair production threshold) and its total reliance on superconducting cavities. An initial test with 56 of these cavities enabled LEP to go to $70 \mathrm{GeV}$ and, although a few snags were encountered, these were quickly rectified. There is full confidence that industry can produce all 272 cavities of the necessary quality, and that $\mathrm{LEP}_{2}$ will meet its target performance.

J. Bordas (Universitat Autònoma, Barcelona) gave an up-to-date status report on the design study for the Barcelona Synchrotron Light Laboratory. This $2.5 \mathrm{GeV}$ third-generation storage ring will use a triple-bend achromat magnet lattice with a circumference of about $250 \mathrm{~m}$. The fully funded design phase aims to present a final report to the Spanish authorities in December 1997. J.-M. Lefebvre (ESRF) explained how careful control of the field quality (phase shimming) in undulators gives an increased beam quality and allows the use of higher photon energy in harmonics. The ESRF now operates with higher beam current, reduced emittance and a very low emittance coupling ratio as a result of refinements to its engineering. Turning attention to protons, S. O'Day (Fermilab, IL, USA) spelled out the ambitious plans to upgrade Fermilab's $1.8 \mathrm{TeV}$ proton-antiproton collider, which will be known as Fermilab III. The goal is to increase the luminosity to $5 \times 10^{31}$ by increasing the beam intensities through a new main injector synchrotron, an upgraded antiproton source and a novel 8 $\mathrm{GeV}$ antiproton recycler ring based on permanent magnets.

\section{Advent of the Factories}

D. Rice (CESR, Cornell) described how the ongoing upgrade of the CESR electron-positron collider will produce a B-factory luminosity of $10^{33}$. This will require superconducting RF cavities (one unit has already been tested in the ring) to support the bunch trains of $500 \mathrm{~mA}$ per beam at $5.5 \mathrm{GeV}$. Associated R\&D is well under way. Impressive progress towards the completion in 1997 of the DAФNE $\Phi$ factory was presented by $\mathrm{G}$. Vignola (INFN, Frascati). The 550 Mev electronpositron linac and accumulator ring is now built and is being commissioned [see EN 27 (1996) 152].

In general, the high beam currents needed for factory-type luminosity presents severe problems for RF systems

\section{Particle Accelerator Prizes}

The EPS Interdivisional Group on Accelerators continued its established practice by awarding two Accelerator Prizes. One prize goes to an individual under the age of 40 at the time of European Particle Accelerator Conference, who has made an original contribution to accelerators within the previous six years. This year's prize, presented at EPAC' 96 by the Chairman of the Prize Committee, G. Rees (Rutherford-Appelton Lab, Chilbolton), went to J. Hangst (Aarhus), shown here on the left. Working at the ASTRID ion storage ring in Aarhus, the prizewinner pioneered the use of laser cooling of bunched stored ion beams, a technique which achieves rapid cooling times and promises ultra-cold dense ion beams in the future.

The other prize goes to an individual or group for achievement and innovation in accelerators within the previous eight years, Awarded to the group headed by

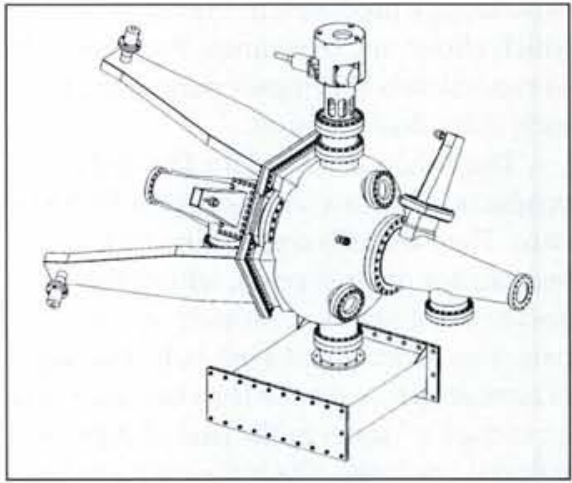

Cleverly designed radiofrequency cavities are needed for accelerators at high-flux particle factories. The sketch illustrates the RF cavity that will be used for the DAФNE $\Phi$-factory being constructed at Frascati. The beam pipe traverses the cavity via the projections on the left-and right-hand sides.

and K. Akai (KEK, Tsukuba) described how these are being solved by feedback systems, advanced coupler designs and Higher Order Mode damping. The parallel approach of minimising the HOM content of RF cavities by clever design was reviewed by R. Boni (INFN, Frascati) and encouraging progress is reported.

\section{Pervasive Synchrotron Light Sources}

The world-wide enthusiasm for synchrotron light remains apparent and G. Dekker (Advance Photon Source, Argonne) reviewed the scene in the USA where consolidation of the recent APS and Advanced Light Source (ALS) facilities is occurring, together with progress in the established sources. H. Kamitsubo (SPring-8, Harima) similarly described the vigorous activity in East Asia, where new facilities are being pursued in China (Shanghai), Japan (Hiroshima, Tohuku, ISSP) and Thailand (Bangkok). For the latter it is suggested to modify and relocate the existing SORTEC $1 \mathrm{GeV}$ ring from Tsu-
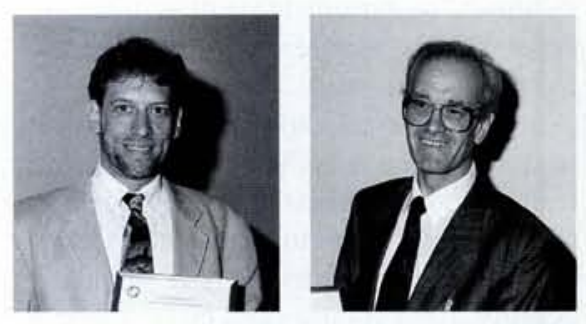

R. Kohaupt (DESY, Hamburg), seen here on the right, for work on longitudinal and transverse feedback systems, it was presented at EPAC' 96 by M. Olivier who chairs the Accelerators Group. By using digital methods of data processing and elements with bandwidths of order $1 \mathrm{GHz}$, the multibunch instabilities limiting the accelerators at the HERA accelerator and elsewhere are successfully counteracted, thereby significantly enhancing the accelerator performance. 
kuba. L. Rivken (PSI, Villigen) compared and contrasted the proposals by several European nations to meet the aspirations of their burgeoning research communities with state-of-the-art facilities, namely DAIMOND (UK), LSB (Spain), SLS (Switzerland), and SOLEIL (France). A source for industrial applications, ANKA (Karlsruhe), has recently received the go-ahead in Germany. Looking beyond the immediate future, the PETRA ring in Hamburg may eventually become available as a 12 $\mathrm{GeV}$ light source providing photons up to $500 \mathrm{keV}$. However, for the ultimate peak brightness, approaching $10^{30}$ in the $\mathrm{X}$-ray region, free-electron lasers in high-energy linacs appear to be the only prospect [see EN 27 (1996) 182].

As a special recognition of the first operation of an accelerator utilising synchrotron acceleration, which took place 50 years ago, T. Wilson (CERN, Geneva) reviewed the story of synchrotrons from 7 $\mathrm{MeV}$ to $7 \mathrm{TeV}$. The $7 \mathrm{MeV}$ energy was attained by Goward and Barnes in London in 1946 with an electron betatron which they had converted to a synchrotron by adding an RF accelerating structure. This was quickly followed by a $70 \mathrm{MeV}$ device at the General Electric Company in the USA, where the first synchrotron radiation was visibly detected. Since then there has been no turning back, and a series of ever more energetic synchrotrons has followed based on ever increasing technical sophistication, including strong focussing, reaching the proposed highest energy of $7 \mathrm{TeV}$ which will be attained by CERN's LHC.

\section{High-Intensity Hadron Accelerators}

The pressing need to reduce the accumulating world stock of plutonium, which results from thermal reactors, was graphically spelled out by C. Bowman (LANL, Los Alamos) and seems only to be feasible by a high-intensity accelerator-based transmutation programme. Although the cost of producing neutrons by accelerators is six times higher than by fission, this differential is greatly reduced in transmuta-

\section{Fifth European Particle Accelerator Conference}

The Fifth European Particle Accelerator Conference (EPAC'96) was held on 10-14 June in sunny (very sunny!) Spain at Sitges, an attractive coastal resort close to Barcelona. More than 780 registered participants from Europe, the USA, Japan and other countries ignored the many attractions of local beaches and instead enjoyed the large and extensive conference facilities of the fully airconditioned Melía Gran Sitges Hotel. The sessions extended over four and a half days in a programme emphasizing excellent review papers and three mini-sessions which reflected particularly interesting accelerator topics, namely: third-generation light sources; linear-collider test facilities (see insert) and superconducting accelerator systems. A special afternoon seminar was devoted to the provision and usage of industrial accelerator services. Through a conscious policy of the Programme Committee to minimize overlap, parallel sessions took place on only two days, and each of the four afternoons accommodated a poster session. To coexist in harmony with the local culture and climate, the conference adopted the Spanish practice of a lunch break from $1 \mathrm{pm}$ and $4 \mathrm{pm}$, with the afternoon sessions continuing until $7 \mathrm{pm}$.

A poster session of over 200 presentations was held on each of the four afternoons of EPAC' 96 . These featured a bewildering display of presentational styles, but all had the same intent, namely to capture the attention of the conference participants who were passing by. That they were successful in this was spoken for by the continual lively activity.

Posters were allocated to sessions according to their technical theme, but additionally, were grouped together by institute so that an impression of the overall work of that institute could be obtained. The best four posters from each afternoon were awarded bottles of excellent Spanish cava and the selection of these caused the conference organizers some difficulty owing to the generally high standard. The only problem was that the posters proved to be such an attraction that attendance in the parallel afternoon sessions was disappointingly low. It is likely that oral poster summaries will not be included at the next EPAC.

The organization of the whole conference ran very smoothly thanks to the Local Organizing Committee, a young team from the Autonomous University of Barcelona under the chairmanship of Ramon Pascual. Intimately connected to the conference business, and vital to the economic success of the meeting, was the exhibition of industries providing accelerator components and associated technology. A total of 34 companies were present and their centrally located exhibition stands were a scene of continuous activity.

The conference banquet was a memorable event held in the atmospheric gothic architecture of Barcelona's medieval naval construction yards. The food and wine were certainly up to the high standards anticipated from the Spanish hosts, but an unexpected surprise for many of the diners was the concluding coffee or "cremat", made to the local fashion. This was an infusion of coffee beans in neat rum!

The conference commenced with a cordial welcome from the Mayor of Sitges who expressed his town's pride in hosting major international meetings of this kind. The Government Minister for Science then noted the importance of accelerator technology to many areas of modern science and referred to the tion by efficiently using the fission neutrons in a chain started by an accelerator This is potentially a huge industry: to deal with present day wastes will require 400 accelerator systems each of order $50 \mathrm{MW}$ beam power. The scale of the problem was provided by T. Broome (RAL, Chilbolton) who described the requirements for highpower targets in spallation neutron sources. The ideal target has high atomic number and melting point, with low corrosion, radiation damage and neutron absorption. Present-day performance is represented by targets at $160 \mathrm{keV}$ (ISIS, Rutherford-Appleton Lab, UK), with the Paul Scherrer Institute in Switzerland commissioning a $1 \mathrm{MW}$ target; clearly, more development is needed.

I. Hofmann (GSI, Darmstadt) reported the initiatives, both in Europe and the USA, for "ignition facilities" to test the ignition of deuteron-tritium pellets using heavy-ion beams. Singly charged heavy ions at about $10 \mathrm{GeV}$ with peak powers of $10-16 \mathrm{~W}$ appear to be the most

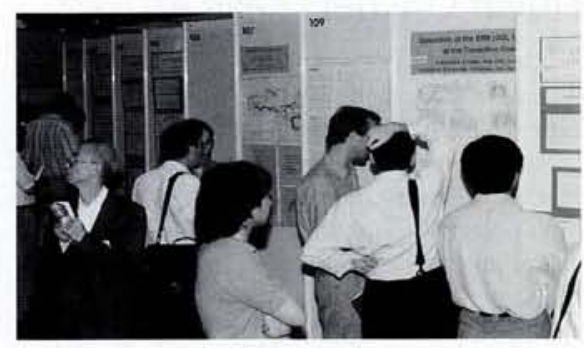

The well-organized EPAC' 96 poster sessions were so popular that attendance at parallel sessions suffered. To gain time, EPAC'98 may not include oral poster sessions.

design study which is presently under way for the construction of an advanced synchrotron light source in the Barcelona region. Michel Olivier (CEA, Saclay), the Chairman of the Conference International Organizing Committee, added his welcome to this fifth conference in the series.

Steve Myers (CERN, Geneva), the Chairman of the Programme Committee, in his pithy resumé of the notable events of the meeting, awarded a special prize to Klaus Wille (DELTA, Dortmund) for his aplomb in continuing his invited talk without pause after falling off the speaker's platform. He also thanked Michel Olivier who is retiring as Chairman of the International Organizing Committee after successfully gestating two EPACs. He is succeeded by Sergio Tazzari (University of Rome) to whom he offered a warm welcome in taking on the arrangements for the next conference in the series, EPAC'98. This will be held in Stockholm on 22-26 June 1998 and the Chairman of the Local Organizing Committee is KarlGunnar Rensfelt (Manne Siegbahn Institute, Stockholm). A cordial invitation to this event is extended to all those interested in the world of accelerators. 
promising route since lower gains are required and a larger fraction of the input beam is recoverable.

Heavy ions also featured in a report by M. Harrison's (BNL, Brookhaven) of the status of the Relativistic Heavy Ion Collider RHIC, which promises entry into a new realm of physics by colliding a spectrum of ion species from protons to Au with energies of $30-100 \mathrm{GeV}$ per amu. All 400 superconducting $4 \mathrm{~T}$ dipoles have been produced but there is insufficient cryocapacity to cool them yet. Heavy ions from the AGS accelerator will be injected into one-sixth of RHIC the ring later this year, but full commissioning is not scheduled until 1999.

\section{Industrial Applications Expanding}

An industrial seminar, chaired by D. Lewis (Amersham International), addressed both the provision and use of industrial accelerator services and showed examples of cyclotrons producing isotopes, low-energy high-current electron linacs irradiating plastics and synchrotron radiation for deep lithography. Additionally, industrial research into materials, catalysts, pharmaceuticals, to mention only a few topics, is being increasingly demanded and catered for by synchrotron radiation facilities. The obvious message is that the industrial application of accelerators is continuing to expand, and is being assisted by a necessary transfer of know-how from research institutes to industry.

\section{EPAC'96 Proceedings on CD-ROM}

More than 1000 abstracts were submitted to EPAC'96. To prepare authors for the eventual fully electronic publishing of the proceedings, these were all produced electronically and sent by file transfer to the EPAC secretariat in CERN. Authors were required during the conference to hand in contributions in electronic format, including all diagrams, on diskette according to a strict timetable. This enabled the hardworking secretariat to check that each paper could be "distilled" successfully into the format in which it would appear in the CD-ROM of the Proceedings, one of the first major conferences in physics to adopt this route. Owing to the carefully thought out details which were given initially to the authors in the shape of document templates and clear instructions, this process went extremely smoothly. By the end of the conference over 800 contributions had been "distilled" to the CDROM file format using the Adode Acrobat software (with a remarkable $70 \%$ success rate at the first attempt) A further 50 documents failed distillation due to a variety of problems. These needed to be corrected before handing all the files to the CD-ROM publisher (the Institute of Physics Publishing) and making them available on the Web at http://www.cern.ch/EPAC. The fully indexed and searchable CD-ROM appeared in September 1996, and a printed version of the Proceedings will also be published by IOPP. It is interesting to note that approximately $25 \%$ of the delegates have requested CD-ROMs in preference to the printed version. A major concern was to make the files compact. It turned out that the average roughly 3.5-page contribution gave a $0.4 \mathrm{MB}$ file, a size which is reasonable for down-loading from the Web.

\section{Linear Colliders have Arrived}

Linear colliders have made great strides during the last eight years or so. The Stanford Linear Collider, the first linear collider, has moved from an accelerator physics experiment to a useful high-energy physics tool where the high polarization of the SLC beams allows precision measurements of certain parameters. At the same time an extensive research effort aimed at a linear collider with an energy around $1 \mathrm{TeV}$ has developed around the world. It is coordinated and reviewed within the framework of a Linear Collider collaboration. There are workshops held each year on specialized topics and an overview workshop, the "LC" series, is held every $11 / 2$ to two years.

The EPAC session on linear colliders began with a review of this world-wide research effort by J.-P. Delahaye (CERN). He presented a concise, but encompassing view of the various approaches to linear collider design. The primary differences in these approaches is the choice of the technology for the linear accelerator used to reach to high energy. In all of the designs the beams are focused to a tiny spot ranging from 5-50 $\mathrm{nm}$. Delahaye's talk was highlighted by a movie produced by G.Guinard (CERN) that illustrated the effect of the accelerator environment on the transverse beam size; it showed graphically how one can accelerate tiny beams in a $30 \mathrm{GHz}$ structure.

After the introductory talk we heard reports from the various test facilities around the world. Hans Braun (CERN) reported on the CLIC Test Facility which tests the twobeam approach where the energy is transferred from a high-current, low-energy beam to a low-current, high-energy beam. This "transformer" is a bit more exotic than the usual ones: the acceleration is done at $30 \mathrm{GHz}$

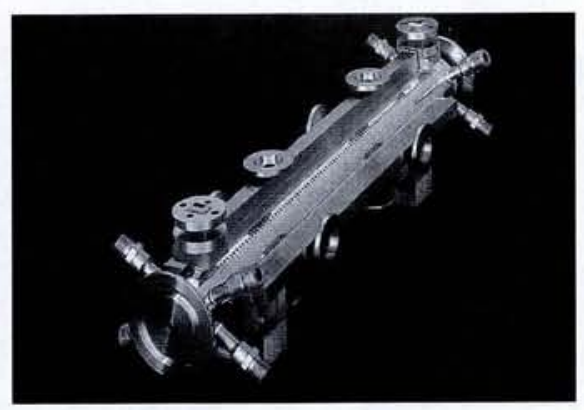

study of the Zero-order Design Report for the NLC, a major milestone for the SLAC effort.

The KEK Accelerator Test Facility was presented by $\mathrm{H}$. Hayano from KEK, Tsukuba, Japan. This facility seeks to test the injectors for a future accelerator, including a special electron storage ring to shrink the beam size to a few microns. The linac is reported to be up and running while the damping ring is being installed now. First tests with beam are planned for early-1997. The KEK Japan Linear Collider (JLC) design also uses $11.4 \mathrm{GHz}$ tech-

An early proptoype of the accelerating section for the main linac of CERN's CLIC collider scheme.

(with accelerator cavities the size of a contact lens) and a acceleration gradient of $100 \mathrm{MV} / \mathrm{m}$, - 5 times higher than the Stanford Linear Collider. Braun showed high-power tests with as much as $100 \mathrm{MW}$ of $30 \mathrm{GHz}$ power and no evidence of breakdown. Accelerating gradients of $90 \mathrm{MV} / \mathrm{m}$ have also been achieved. The next phase at the CLIC Test Facility will include a test of the two-beam concept. The drive beam will be deaccelerated and and the resulting 30 $\mathrm{GHz}$ power will be used to accelerate a low intensity beam.

R. Ruth (Stanford Linear Accelerator Center, CA, USA) presented the Next Linear Collider Test Accelerator (NLCTA) which uses 11.4 $\mathrm{GHz}$ technology, four times the frequency of the SLAC linac. This facility is a $0.5-1 \mathrm{GeV}$ model of the high-gradient linac for the Stanford design. Ruth showed data from the klystrons, RF pulse compression system and accelerator structures that exceeded the NLC design specifications. Acceleration gradients of $65-120 \mathrm{MV} / \mathrm{m}$ have been achieved in highpower tests. The NLCTA RF system is being installed now and this test accelerator will have first beam in late-1996. Ruth also noted that SLAC has just completed a feasibility nology but a small contingent at KEK has been investigating $5.7 \mathrm{GHz}$.

Work at DESY, Hamburg, towards a future collider was described by B. Aune (DESY) who reported on the TESLA Test Facility which seeks to accelerate beams at 15 $\mathrm{MV} / \mathrm{m}$ up to $0.5 \mathrm{GeV}$. The TESLA linear collider design uses the efficient energy transfer in a superconducting accelerator to obtain very high beam power. This allows a somewhat larger spot size at the final focus. Aune showed tests of accelerating structures achieving $25 \mathrm{MV} / \mathrm{m}$, the design goal for the TESLA linac. N. Holtcamp (DESY) then presented work on the S-Band Linear Collider Test Facility. This design uses the RF technology developed for the SLAC linac more than 30 years ago. He showed examples of the tunnel layout and emphasized differences in the new S-band design, higher-power klystrons and tighter structure tolerances. It is planned to be complete next year both DESY test facilities.

Overall the linear collider session showed the field's impressive progress. With the completion of the test facilities and extensive design studies, linear collider technology has matured to the point that in the very near future one can consider the construction of 0.5 $\mathrm{TeV}$ linear collider which could be upgraded to $1 \mathrm{TeV}$ and beyond.

R. Ruth, SLAC 